\title{
Cultura mapuche: un antiguo ideal de persona para una nueva historia
}

\section{Mapuche culture: an ancient ideal of the individual for a new history}

Aceptación: marzo 2008.

Aprobación: mayo 2008.

Ramón Curivil Paillavil'

\section{RESUMEN}

En el ensayo sintetizo un trabajo de investigación financiado por Missio Aachen, Alemania, y que se realizó entre el año 2007 y 2008 en una zona que abarca tres comunas: Saavedra, Carahue y Nueva Imperial, en la IX región de la Araucania. El propósito del proyecto fue recopilar y sistematizar información sobre: idea de hombre o idea de persona, maneras de ser, de vivir y convivir en el mundo y con el mundo. A partir de esas recopilaciones comparto algunas reflexiones en este ensayo.

Palabra clave: Idea de hombre o idea de persona, maneras de ser, de vivir y convivir en el mundo y con el mundo.

\section{ABSTRACT}

In this essay I summarise a research work financed by Missio Aachen, Germany, and carried out between 2007 and 2008, in a zone covering three districts: Saavedra, Carahue and Nueva Imperial, in the Araucania Region. The object of the project was to collect and systematize information on: idea of man or idea of person, ways of being, living and co-existing in the world and with the world. In this essay I share some of the reflections provoked by the information collected.

Key words: Idea of man or idea of person, ways of being, living and co-existing in the world and with the world.

Ramón Fco. Curivil Paillavil. Profesor de Filosofía. Magister en Ciencias Sociales. Docente del Complejo Educacional Claudio Arrau León, comuna de Carahue, Coinvestigador del Centro de Estudios de la Realidad Contemporánea (CERC UAHC) pijumajin@hotmail.com 


\section{Presentación}

En este artículo doy a conocer un trabajo de investigación sobre "Imagen de ser humano o concepto de hombre en la cultura mapuche". El diseño metodológico combinó técnicas de observación participante en actividades de carácter sociocultural que realizan las organizaciones, como participación activa en xaun (reuniones comunitarias y de dirigentes), nvxam (diálogo) con jóvenes y personas de edad. En este sentido se trata de una investigación exploratoria y eminentemente cualitativa.

El supuesto que guía la investigación es el siguiente: "existe una manera original de ser humano de acuerdo a la cultura mapuche, que tiene que ver con una relación respetuosa y armónica no solo con la Madre Tierra (Ñuke Mapu), sino también con todos los seres vivos (fij mogen)". Estas ideas de ser humano, muestran claramente la tensión entre un pasado (kuifi) y un aquí y ahora (fau, feula), donde la vida mapuche se desarrolla bajo la influencia de dos corrientes de pensamientos: el pensamiento occidental cristiano y el pensamiento mapuche, sin que necesariamente se dé aún la sintesis cultural y filosófica.

Las reflexiones surgidas a lo largo de la investigación las presento como un ensayo y tienen el propósito de crear puentes para la comunicación entre culturas, específicamente para el diálogo sobre distintas concepciones humanas y de esta forma - parafraseando al filósofo Raúl Fornet-Betancurt- conocer la riqueza de las razones con que el pensamiento mapuche da razón de su vida².

La estructura del artículo se ha ordenado de la siguiente forma: una reflexión teórica preliminar respecto de los principales conceptos involucrados en la investigación; en segundo lugar, algunos aspectos relevantes de la investigación en relación a una manera de entender la tierra, aún vigente, en un sector importante de la población mapuche (el sector que valora más la tradición), frente a una mentalidad moderna avasalladora, de apropiación y destrucción de la Madre Tierra; en tercer lugar, algunas reflexiones sobre el antiguo ideal de persona presente en el imaginario mapuche, donde es posible percibir elementos de la tradición cristiana; y cuarto, una reflexión final que deja abiertas las posibilidades para un diálogo que permita nuevas interpretaciones.

\section{Reflexiones teóricas preliminares}

El concepto de cultura ha sido ampliamente discutido por los científicos sociales ${ }^{3}$, independiente de la acentuación que podamos hacer, las diversas posturas epistemológicas tienen en común el entender la cultura como: a) un sistema compuesto por patrones de comportamiento aprendidos que favorecen la adaptación de las comunidades humanas a sus respectivos ambientes; b) el modo particular como los hombres cultivan su relación con el mundo, con la naturaleza, entre sí mismos, con sus antepasados y con la divinidad. Asi entendida la cultura, esta abarca la totalidad de la vida, el conjunto de valores que lo animan y de disvalores que lo debilitan. En este sentido podemos decir que "cada cultura va conformando un determinado estilo de vivir y ver las cosas y una determinada forma de sentir, gustar y apreciar la vida y sus contingencias..." 4 . El concepto mapuche más cercano a esta noción de cultura es mapucemogen, que literalmente significa "estilo de vida mapuche". Se trata de una forma de vida donde el "kuifi" (antes) es imagen y representación de un estilo de vida que se ha logrado plasmar

\footnotetext{
Cf. Transformación intercultural de la filosofia, p. 14.

Desde la antropología, la cultura "... es conjunto complejo, que abarca los conocimientos, creencias, el arte, el derecho, la moral, las costumbres y los demás hábitos y aptitudes que el hombre adquiere en cuanto miembro de una sociedad"... (E. Tylor: Primitive Culture, Londres, 1871). Desde la sociología, "es un conjunto estructurado de maneras de pensar, sentir y obrar más o menos formalizadas que, aprendidas y compartidas por una pluralidad de personas, sirven, de un modo objetivo y simbólico a la vez, para constituir a esas personas en una colectividad particular y distinta"... (Rocher Guy: Introducción a la sociología general. Herder, Barcelona, 1973, pp. 111-116). Mientras que desde la antropología filosófica, "la cultura es una trama de significaciones..." (Cf. Clifford Geertz, en: La interpretación de las culturas, p. 20).

$4 \quad$ Parker Cristián: Cultura, en: Boletín de filosofía $N^{\circ} 9$, p. 96.
} 
en el pasado (kuifimogen) y que hoy ilumina el presente, el aqui y ahora (fau, feula).

En el lenguaje cotidiano de las personas que han tenido acceso a una mayor formación profesional y académica, lo antiguo y tradicional tienen en general una connotación negativa, ya que para ellos el pasado carece de valor. En este sentido las culturas indigenas son consideradas como culturas arcaicas que no tienen nada que aportar a un mundo moderno, donde lo moderno representa lo nuevo, lo actual, lo que tiene valor. Estas ideas han sido difundidas incluso por algunos pensadores latinoamericanos, entre los cuales se puede mencionar el escritor peruano Mario Vargas Llosa, quien justifica la desaparición de los indigenas cuando afirma que "tal vez no hay otra manera realista de integrar nuestras sociedades que pidiendo a los indios pagar ese alto precio; tal vez, el ideal, es decir, la preservación de las culturas primitivas en América, es una utopía incompatible con otra meta más urgente: el establecimiento de sociedades modernas en las que las diferencias sociales y económicas se reduzcan a proporciones razonables, humanas, en la que todos puedan alcanzar al menos una vida libre y decente..." ${ }^{\prime 5}$. Sin embargo, desde la perspectiva mapuche, el kuifi, la tradición, el pasado, la experiencia de vida acumulada (kuifimogen), representa un ideal de vida, sin la cual no es posible una continuidad y permanencia en el tiempo. Por eso, la no valoración de la tradición en la cultura moderna demuestra que existe una gran dificultad en imaginar un mundo, de carácter plural, capaz de articular la diversidad cultural, hecho que demuestra que la modernidad es por naturaleza agresiva, violenta y monocultural, que busca imponer un estilo de vida con miras a implantar una civilización mundial que monetariza todas las dimensiones humanas ${ }^{6}$, poniendo en peligro la identidad de los pueblos y naciones indigenas.
Respecto al concepto de identidad, considero necesario ensayar nuevos enfoques epistemológicos a partir de una filosofía intercultural, que parta del diálogo, el reconocimiento y la aceptación de la diversidad cultural y religiosa. En la práctica esta opción representa el único camino de acercamiento entre representantes de culturas diferentes. Hasta ahora el camino ha sido inverso, ya que históricamente la sociedad mayoritaria (occidental cristiana) ha optado por negar sistemáticamente la identidad del "otro" en cuanto indígena, olvidando que "mientras menos se conoce a una persona o a un grupo humano con el cual se convive, mayores son los prejuicios que sobre ellos se pueden concebir y mayor la violencia que se puede desatar. Negarse a conocer al otro, su vida, sus intereses, sus esperanzas, es negarse a reconocerle su dignidad y sus derechos como persona o como comunidad humana..." . Hoy debemos considerar nuevas formas de acercamiento teniendo presente que la identidad "se moldea en parte por el reconocimiento o por la falta de éste; a menudo también por el falso reconocimiento de otros, y asi, un individuo o un grupo de personas puede sufrir un verdadero daño, una auténtica deformación si la gente o la sociedad que lo rodean le muestran, como reflejo, un cuadro limitativo, o degradante o despreciable de sí mismo. El falso reconocimiento o la falta de reconocimiento puede causar daño, puede ser una forma de opresión que aprisione a alguien en un modo de ser falso, deformado y reducido...". . De la misma forma podemos afirmar que "... el falso reconocimiento no solo muestra una falta del respeto debido. Puede infligir una herida dolorosa, que causa a sus víctimas un mutilador odio a sí mismas. El reconocimiento debido no solo es una cortesía que debemos a los demás: es una necesidad humana vital"...9 Esta necesidad de reconocimiento lo podemos ver claramente en las actuales demandas del pueblo mapuche que

\footnotetext{
Citado por Antillanca y Loncon: Entre el mito y la realidad, pp. 45-46.

Cf. Transformación intercultural de la filosofia, p. 340.

Diaz F. José Fernando: El proyecto hístórico del Pueblo Mapuche, p. 29.

Taylor Charles: El multiculturalismo y la politica del reconocimiento, p. 44.

Idem.
} 
se centran fundamentalmente en el tema del reconocimiento. Estas demandas por el reconocimiento y la valoración de las diferencias no debe ser interpretado como un proceso nostálgico de indígenas que quieren volver al pasado. Muy por el contrario, se trata de una demanda permanente e histórica, que debemos entender bajo el principio de que el deseo de mantener las diferencias es "asumir el riesgo de existir"10, de ahí el esfuerzo de un sector de mapuches por recuperar el territorio, potenciar y no perder la lengua, los ritos, los relatos, las artes, las vestimentas. Esto no es una pretensión de quedarse en el pasado o no querer avanzar con la historia, el tiempo histórico avanza irremediablemente. En esta dinámica de mantener lo propio como elemento identificador, el concepto mapuche más cercano a identidad es el de az mapuce, que hace referencia a un rostro, un carácter y una manera de ser persona, sea hombre o mujer. Esta manera de ser original se ha diluido en el tiempo como consecuencia de un proceso colonizador llevada a cabo por el Estado chileno. Es decir, existe en la sociedad chilena una imagen negativa ${ }^{11}$ de mapuche y de indigena que se ha construido intencionalmente y que hace mucho daño a las nuevas generaciones. Se trata de algo pensado, elaborado racionalmente, por lo que en cierta forma es posible cambiar mediante políticas sociales y educativas oficiales, donde se fomente el valor y el respeto a la diversidad ${ }^{12}$. Pero sin duda lo más complejo es el hecho de que en la sociedad chilena también existe una imagen negativa en el "inconsciente nacional", que se transmite mediante prejuicios y estereotipos aprendidos principalmente en el hogar, lo que resulta más difícil de extirpar.

\section{Aspectos relevantes de la investigación}

"Xekan meu, zugun meu, kvzau meu, tukutun meu kimgekei mapuce wenxu, mapuce zomo..." (Domingo Coña-Pillumallin).

En la forma de caminar, de hablar, en la forma de trabajar y de vestirse, se conoce al hombre y a la mujer, si verdaderamente es mapuche..."

En relación a los resultados de la investigación, en lo medular el proyecto recabó información sobre la manera de entender la tierra, maneras de ser, de vivir y convivir en el mundo y con el mundo; memorias del relato mítico del Xen Xen y Kai Kai filu; el antiguo ideal de hombre o idea de persona en el imaginario mapuche. Sobre estos aspectos comparto algunas reflexiones "provocativas" que permitan discutir una nueva imagen de mapuche a partir de "un antiguo ideal de persona para una nueva historia..."13.

Una filosofía de la tierra: "Nosotros pertenecemos a la tierra".

Comienzo con la siguiente pregunta: ¿cómo pensar nuestra humanidad, nuestro ser persona, a partir de la sabiduría tradicional acerca de la tierra?

La siguiente reflexión es iluminadora:

"El mapuche le tiene cariño a la tierra porque somos de la tierra, dependemos de la tierra... dueños de la tierra, uno aunque viva mal pero es su tierra... uno está aclimatado con su terreno aunque sea poquito... Nosotros pertenecemos a la tierra

10 Certeau, citado por Diaz Fernando, en op. cit. p. 40.

11 Las actuales politicas indigenistas llevadas a cabo por el gobierno de turno y aplicadas especificamente al sistema educacional han ido cambiando lentamente esta imagen negativa del pueblo mapuche.

12 Aunque para el pueblo mapuche el paso más importante es lograr el reconocimiento de sus derechos políticos como pueblo, porque el reconocimiento forja la identidad, particularmente en su aplicación fanonista, en el sentido de que los grupos dominantes tienden a afirmar su hegemonía inculcando una imagen de inferioridad a los subyugados. Cf. Taylor Ch. op. cit., p. 97.

13 La idea de una nueva historia la extraigo de Armando Marileo, quien "ha planteado la idea de que la sociedad mapuche ha transitado hasta ahora por cuatro ciclos históricos: la primera historia sería aquella que va desde la creación de la especie humana mapuche hasta la gran batalla de Xen Xen y Kai kai...; la segunda historia comienza en el momento de la lucha entre estas fuerza y que culmina con la invasión española...; la tercera historia, va desde los tiempos de la invasión hispana hasta los momentos de mayor expresión de la dominación de los estados nacionales chileno y argentino; la cuarta historia, es el presente y tiene como referente el proceso de reconstrucción de la sociedad mapuche...". Citado en: i... Escucha Wigka...!, p. 24. 
porque vivimos en armonia con ella. Mapu significa tierra y che gente o persona. Nosotros pertenecemos a la tierra porque la tierra nos cobija cada día y decimos que nosotros afloramos desde la tierra porque nuestra concepción de tierra, según nuestros antepasados, es que todos nacemos de la tierra y eso significa mapuche... y vivimos de acuerdo en armonía con ella con el cosmos mismo. Todo lo que vemos, todo lo relacionamos a la tierra, la fuerza que viene de la tierra... Para el maremoto del año 60 nuestros antepasados hicieron oración, porque dijeron que la tierra estaba castigando a los mapuches y por eso se produjo el terremoto y por eso, la gente se acercó a pedirle a la tierra para que se tranquilizara..." (Tránsito Segundo LipanLlarquenco-).

Ser de la tierra, pertenecer a la tierra es muy distinto a sentirse dueño, amo y señor de la tierra. Esta diferencia se ve claramente al comparar la mentalidad mapuche con la mentalidad moderna de Occidente.

En Occidente, a partir del siglo XV en adelante, se produce un cambio de mentalidad respecto de la naturaleza, dado que a partir de ese periodo la tierra es tratada como objeto que se tranza en el mercado. Este cambio de mentalidad es resultado de la influencia del racionalismo cartesiano y del empirismo lockeano. El maestro de la duda metódica es el mejor representante de esta separación entre el hombre y la tierra o, dicho de otra forma, entre "sujeto-objeto". Para René Descartes, el sujeto hombre no es más que "res cogitans", es decir, una cosa que piensa, que duda, que entiende, que afirma, que niega, que quiere y que carece de materialidad. Dice, por ejemplo, "... examinando atentamente lo que yo era y viendo que podía suponer que carecía de cuerpo y que no habia mundo, ni ningún lugar en que yo estuviese; pero que no por esto podía suponer que yo no existia, sino que, al contrario, por lo mismo que dudaba de la verdad de otras cosas se deducía muy evidente y ciertamente que yo existia, mientras que si yo hubiese dejado de pensar, aunque todo lo demás que hubiere imaginado alguna vez hubiera sido cierto, no tendria ninguna razón para creer que yo había existido, deduje que yo era una sustancia cuya naturaleza o esencia era pensar, y que para ser no necesitaba ningún lugar ni dependía de ninguna cosa material, de modo que este yo, es decir, el alma, por la que soy lo que soy, es enteramente distinta del cuerpo, y aún más fácil de conocer que él, y aunque el cuerpo no existiera, ella no dejaría de ser todo lo que es"14. Esta idea de ser humano, en el fondo genera la ilusión de que un sujeto puede existir sin el cuerpo y que "puede seguir actuando racionalmente como si no pasara nada aun cuando la tierra la esté destruyendo eficientemente con su explotación racional"15. De la filosofía cartesiana, finalmente podemos deducir que no necesitamos la tierra para existir y lo que le hagamos a la tierra no se la hacemos a los hijos de la tierra, como por el contrario nos recordara el jefe indio norteamericano Seatle.

Si con René Descartes se produce el distanciamiento del hombre respecto de la naturaleza porque ya no se siente parte de la tierra, con lo cual reniega de su condición de hijo de la tierra, con John Locke desaparece la Madre Tierra, como propiedad comunitaria para dar paso a la propiedad privada y de esta forma llevarla al mercado. En su obra Segundo tratado sobre el gobierno civil, sostiene que la tierra, sus criaturas y sus productos pasan a ser meros objetos del derecho de los hombres, desentendiéndose de cualquier dependencia respecto al bien común.

En relación a una hipotética situación originaria, Locke comienza su reflexión diciendo: "Dios que ha dado en común el mundo a los hombres, les ha dado también la razón, a fin de que hagan uso de ella para conseguir mayor beneficio de la vida, y mayores ventajas. La tierra y todo lo que hay en ella le fue dada al hombre para soporte y comodidad de

14 René Descartes: Discurso del método. Cuarta parte, p. 65.

15 Juan Antonio Senent de Frutos: "La tierra y la naturaleza en el horizonte de la subjetividad moderna", en: El lugar de la tierra en las culturas. Un diálogo de cosmologias ante el desafío ecológico, p. 167. 
su existencia. $Y$ aunque todos los frutos que la tierra produce naturalmente, así como las bestias que de ellos se alimentan, pertenecen a la humanidad comunitariamente, al ser productos espontáneos de la naturaleza; y aunque nadie tiene originalmente un exclusivo dominio privado sobre ninguna de estas cosas tal y como son dadas en el estado natural, ocurre, sin embargo, que como dichos bienes están ahí para uso de los hombres, tiene que haber necesariamente algún medio de apropiárselos antes de que puedan ser utilizados de algún modo o resulten beneficiosos para algún hombre en particular" (\& 26) ${ }^{16}$. Pero como es necesario que estos bienes se multipliquen y rindan, deben ser apropiados individualmente. De esta forma el concepto de tierra comunitaria "soporte de la vida común de cada pueblo y por tanto algo que pertenece a los comuneros, y de la que estos no pueden disponer o enajenar, ni tampoco pueden enajenarse de ella en tanto que también pertenecen a la tierra, es decir, se trataba de una mutua posesión, de una posesión en reciprocidad. Pasa a ser considerada, no ya como terra mater, lugar de nutrición y de identidad como en las sociedades también europeas, sino como terra nullius, tierra de nadie" ${ }^{17}$. En la filosofía de Locke, "algo común" significa que puede ser apropiable por cualquiera y ello sin depender del consentimiento de nadie. Incluso con una argumentación religiosa justifica la apropiación de los bienes comunitarios dado que el derecho positivo está por sobre el derecho natural. Dios "Ha dado el mundo para que el hombre trabajador y racional lo use, y es el trabajo lo que da derecho a la propiedad, y no los delirios y la avaricia de

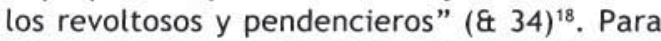
Locke es claro que el hombre trabajador y racional es el europeo (la parte civilizada de la humanidad) y los revoltosos y pendencieros son los miembros de las sociedades tradicionales, y como sus bienes no están protegidos por ningún sistema de derecho positivo, las tierras comunitarias son tierras de nadie (res nullius), apropiable por cualquiera.
Del pensamiento de Locke podemos llegar a la siguiente conclusión: el derecho de propiedad privada da derecho al disfrute, incluso al uso y al abuso de las cosas poseídas, y si se destruyen las cosas poseídas, supuestamente no se destruye el poseedor. En otras palabras, si ya conseguimos dominar la naturaleza (la tierra), definitivamente no dependemos de ella, y aunque la destruyamos, el hombre no se destruye a sí mismo. De esta forma es claro que el pensamiento moderno, que tiene su cuna en Europa, prescinde de las condiciones de sobrevivencia, dado que esta mentalidad llevada al extremo se ha transformado en un verdadero saqueo a la naturaleza, que se expresa como contaminación y cambio climático que pone en peligro la sobrevivencia humana en el planeta.

Contrario a este pensamiento, en el largo caminar de su historia, el pueblo mapuche aprendió una relación diferente con la tierra. $Y$ esto lo podemos ver claramente en el testimonio de nuestros dirigentes. Se trata de una relación respetuosa, porque "pertenecemos a la tierra". Ella es productora de vida $y$, en este sentido, es nuestra Madre Tierra (Ñuke Mapu).

De acuerdo al pensamiento mapuche (mapuce rakizuam) la vida existe de diversas formas (fij mogen mvlei taci mapu meu): se manifiesta en nosotros como personas, seres humanos (ce), pero también se manifiesta como un espíritu (pvjv), como un animal ( $k u$ jiñ), como un pájaro ( $v \tilde{v} v m)$, como una serpiente (filu), etc., pero todos dependemos de la Madre Tierra. Ella nos cobija, nos alimenta y nos enseña a vivir y a convivir en la diversidad. Ella distribuye los espacios de vida y en cada lugar deja alguien como protector y vigilante (los geh). Estos son seres inmateriales que pueden traspasar las fronteras de la naturaleza y viven en una montaña, en un volcán, en los ríos, en los lagos, en los mares y en los montes, etc. Estos lugares son considerado espacios sagrados porque tienen newen (fuerza y poder).

16 Citado por Senent, en op. cit. p. 169.

17 Op. cit., p. 170.

18 Citado por Senent, en op. cit., p. 173. 
Este pensamiento acerca de la tierra es al mismo tiempo una religión, la religión de la tierra ${ }^{19}$, dado que existe la convicción de que la Madre Tierra tiene newen (fuerza, poder, energía) y en ese sentido no es un objeto que podamos destruir. El hombre no destruirá la tierra, pero con su actuar irresponsable hará de ella un desierto inhabitable.

De acuerdo a la sabiduria de los ancianos (kuifikece), existe una convicción religiosa de que:

"... mapu ta kiculelai... mvlei ta gehmapu, welu pu geh kicugvneukvlelaigvn tati..." (Catalina Paillavil-Pillummallin).

"... la tierra no está sola... Ella está protegida por los geh, pero ellos no se mandan solos..."

Los geh preferentemente están en los xayenko y menoko ${ }^{20}$ :

"El xayenko es una vertiente de agua... el dueño del agua dicen que es un geh ko, yo lo he escuchado, pero no se por qué habrá eso... Todas las aguas dicen que tienen un geh. Antes le pedian permiso para sacar agua, para que esa agua saliera sana y no haga daño... yo recuerdo cuando limpiábamos el pozo, nos decian, ustedes no maten a los camarones ni a los gusanos, porque esos son los geh ko. Todas las aguas tienen geh ko... De la misma forma geh mapu hay en todas partes, igual que el dueño del agua. Por ejemplo, en una quebrada si uno limpia debe pedir permiso, si no en la noche se sueña con algo. Yo creo que en todas las quebradas existe el geh... A los geh hay que tenerle respeto. Le voy a contar un caso, yo tuve un hermano, una vez mi mamá le dijo: jno limpies ahí porque hay un geh mapu! Y uno como joven no les hace caso a los mayores. Él fue a limpiar y des. pués quemó y en la noche no podía dormir, dijo que estaba desesperado. Y mi mami le preguntó ¿qué hiciste?.. ¡No ve! Debes pedir perdón. Debes ir a rezar. No ve el geh mapu está en todas partes, no en una sola parte..." (Pedro Curifuta-Curileufu)

Esta mentalidad religiosa tiene consecuencias prácticas en la vida y es parte de la riqueza espiritual que permite un trato religioso con la naturaleza. Por ejemplo, en la vida diaria, toda actividad que realiza el hombre (ce), sea que trabaje la tierra, sea que se bañe en el río, sea que decida matar un animal, debe hacer primero un gejipun, es decir, dialogar, pedir, agradecer, hacer algún gesto de comunión (micaun) y agradecimiento a la Madre Tierra. El mismo gesto se realiza ante la divinidad (Dios) en una ceremonia de gijatun, como se expresa claramente en el siguiente testimonio:

"... mvlemvlegele gijatun aflayai taiñ zugu, aflayai taiñ mogen. Si rogamos al cau Dios aflayai tati... a diosito hay que darle, le dan mudai, lagvmgekei kujiñ fei ñi elel-kai. Después ta zoi eluen feipigekei, kaxvlelayu kuyiñ, micayayu pigei cau Dios gijatunmu... micagei ta cau Dios, gijatunmu feimu elugekei mudai ka iael, cruz mu, fei zoi wvli fvca cau, wenu cau, zoi kvme suertekei ce... Fenxen ce xaukei gijatun mu, feula hasta pu wigka ayvkei gijatun pu cau mapuceukvleigvn..." (Rosa Porma-Ranco).

"... mientras haya gijatun, lo nuestro, nuestra forma de vida no se acabará. Si rogamos al Padre Dios, 10 nuestro no se acabará... por eso, a diosito hay que darle mudai, matar animales porque todo eso lo ha dejado él. Después se le pide para que nada nos falte, se le dice, elegiré un animal para ti, para que lo compartas con nosotros y seamos mica ${ }^{21}$. Así se le dice

19 Tema que abordo en el libro "La religión de la tierra. Una herencia de nuestros antepasados". Ver bibliografia.

20 En la concepción de mundo mapuche toda la tierra tiene newen (fuerza, energia, poder), sin embargo, hay lugares como el gijatuwe, los xayenko, los menoko, incluso un árbol o una piedra, donde en cierta forma se concentra parte de este poder personificados en los geh. En estos lugares es necesario hacer un gejipun, es decir, dialogar, hacer un ruego o petición o pedir permiso.

21. Mica (se pronuncia suavemente micha como en castellano), es un grado de amistad y confianza, que permite compartir la comida y comer de un mismo plato. De esta forma se crea un clima adecuado para pedir y solicitar algún favor. 
a Dios en el gijatun. Hay que compartir con él y eso se hace dándole mudai y carne al pie de la $\mathrm{cruz}^{22}$, asi él se pone más generoso y uno tiene más suerte... Mucha gente se reúne en la ceremonia del gijatun. Ahora hasta los wigka son como los mapuches...".

Esta filosofía acerca de la tierra tiene consecuencias trascendentales para asegurar la continuidad de la vida en la Madre Tierra.

Una nueva humanitas ${ }^{23}$ a partir del mito del Xen Xen y Kai Kai filu

En los nvxam (diálogo) con los kuifikece (personas antiguas) y los kimce (sabios) acerca del newen (energía, fuerza, poder) que tiene la tierra, aparece reiterativamente el recuerdo de una catástrofe ${ }^{24}$ de carácter universal semejante al diluvio judeo cristiano.

Aquí comparto parte de algunos relatos, seleccionando dos versiones recopiladas en la zona de Puerto Saavedra.

\section{Kuifi, fvxa kuifi}

(Antes, hace mucho tiempo)

1. "Kuifi dicen, fvxa kuifi, hubo una avenida. Salió el lafken, decía mi abuelita y casi terminaron todos los mapuches.

2. Dicen que cuando salió el mar la gente se iba en el Xen Xen... cuando salía el mar el Xen Xen subía arriba, va creciendo va creciendo y la gente dicen que se subia al Xen Xen...

3. Dicen que en el Xen Xen había cualquier bicho, culebra, de todo.

4. Antes, mucho antes, eso conversaba mi abuelita.

5. Decia que hubo una salida de mar y dicen que se salvaron dos hermanas casadas y dicen que de ahí se rindió la gente, se rindió la familia otra vez...
6. Pero cuando decian ¡Ayyy!, se caían al agua, decía mi abuelita. Pero cómo no iban a tener miedo, digo yo, si habia tantos bichos en el Xen Xen...

7. Por eso solo dos hermanas casadas vivieron y ahi se rindió la familia. Eso contaba mi abuelita. Yo era niñita...

8. Decía, cuando el mar llegaba el Xen Xen subía y de esas dos hermanas se rindió la gente..."

(Rosa Porma - Ranco).

\section{Y sacrificaban a los parawuay}

1. "Mi abuelita contaba que los niñitos que se criaban huachitos, que le decian parawuay, le cortaban la cabeza y lo tiraban al agua para que se calme el mar.

2. Eso pagaban al mar los mapuches, antes. Sacrificaban los niños que no tenian papá, para que se calme el mar y vuelva a su lugar...

3. Al final dicen que se salvaron todos los que estaban en el cerro...

4. Así como bajaban las aguas del mar, bajaba el cerro también, los que resistieron llegaron vivo, los otros murieron...

5. De los sobrevivientes volvió a crecer la familia de nuevo...

6. Eso lo que contaba mi mamá: crecía y se multiplicaba la familia nuevamente con los sobrevivientes, decía...

(Zuñilda Tranamil Dollinco).

¿Qué aparece en estos dos relatos? La expresión "Kuifi, fvxa kuifi" nos lleva no al inicio de los tiempos, sino al término de la primera historia mapuche. El propósito del mito no es responder a la pregunta ¿cuál es el origen de los mapuches? El relato sí entrega algunas pistas respecto de ¿por qué ocurrió este fenómeno de la naturaleza? En ese tiempo podemos suponer que algo malo ha ocurrido entre los mapuches que causa la

22 Desde la parte norte de Puerto. Saavedra hasta Galvarino, una cruz reemplaza al rewe casi en todos los gijatuwe.

23 La humanitas entendida como "proceso de formación de la existencia humana". Cf. Transformación intercultural de la filosofía, p. 311.

24 Prácticamente todas las comunidades visitadas tienen un cerro Xen Xen como lugar de refugio en tiempos inmemoriales, cuando hubo una gran inundación. Debido a la influencia del cristianismo, la mayoría de las comunidades relaciona esta inundación con el Diluvio bíblico. 
furia de la Madre Tierra, el enojo de la naturaleza. Por lo tanto, "alguien" decide acabar con los mapuches. Se trata claramente de un castigo no individual, sino colectivo. El encargado de exterminar a los mapuches no es Dios ni los dioses, sino el poder, la fuerza del mar. Es la naturaleza que se rebela contra el hombre. Este poder toma la forma de un monstruo marino, especificamente una serpiente llamada Kai Kai que moviliza las aguas del mar, arrasando con todo lo que encuentra a su paso. Ante esta amenaza de destrucción total, todos los seres vivos buscan refugio en un cerro Ilamado Xen Xen. El Xen Xen es un lugar poderoso que crece en la medida que suben las aguas. Tanto el cerro Xen Xen como la serpiente Kai Kai representan dos poderes en pugna. Un poder destructor (el mar) que provoca un caos total. El agua, en este caso, es simbolo de muerte ya que destruye todo lo que encuentra a su paso; y un poder salvador que se encuentra en un cerro, al que acuden desesperadamente todos los seres vivos, incluyendo los bichos (seres insignificantes).

El que se enoja y decide terminar con los mapuches no es Dios ni los dioses, sino el poder de la naturaleza que vive en medio de las aguas y que está representado en un monstruo marino. Pero se trata de un poder que se puede controlar y neutralizar mediante un sacrificio (Segundo relato). Este sacrificio, para calmar la furia del mar, no fue un ser vivo cualquiera, fueron seres humanos que se sacrificaron. El parawuay es un hijo natural, que en el relato representa la indefensión plena. El parawuay es un desvalido y desprotegido total. En el fondo, eso somos ante una realidad catastrófica. Por lo tanto, ese es el sacrificio agradable que satisface plenamente al poder del mar.

Después del caos (destrucción) viene la calma y la vida empieza de nuevo. Se inicia un nuevo ciclo de la vida y de la diversidad. Todo empieza de nuevo. La vida humana "se rin- de", se multiplica a partir de dos parejas. Y de esta forma se inicia una segunda historia. Ambos relatos marcan claramente el término de la primera historia y el inicio de una segunda historia, porque "De los sobrevivientes volvió a crecer la familia de nuevo...".

El relato no dice que somos obra de los dioses o que los dioses están detrás del castigo, sin embargo, podemos deducir lo siguiente: primero, compartimos una misma naturaleza con el resto de los seres vivos, y ante una amenaza de la naturaleza (maremotos, cambio climático en general) corremos la misma suerte; segundo, la tierra es nuestra Madre y la diversidad de vida depende de ella, porque todos somos hijos de la tierra; tercero, si algo se insinúa en el relato respecto de nuestra humanitas, es que la tierra siempre da una posibilidad para que la existencia humana continúe; y cuarto, somos seres en relación cuya principal caracteristica es nuestra capacidad de raciocinio y este hecho nos da una mayor responsabilidad ante la naturaleza, porque sabemos que si el hombre no respeta y destruye la naturaleza, como lugar de la vida, finalmente se destruye a si mismo.

Muchos mapuches y no mapuches hacen una interpretación bíblica del Xen Xen, sin embargo, lo más interesante es que los kuifikece (ancianos) hacen también una reinterpretación de la vida actual, y en lenguaje cristiano dicen que "diosito ya no nos escucha porque estamos demasiado cristianizados 25 ":

"... Pero yo diría que mvte wigkaukvleiñ peñi fei mu ajkvlai cau Dios (Dios ya no nos escucha porque hemos asumido las creencias wigka) ...Digamos la verdad, Dios ya no nos quiere porque rume winjaukvleiñ ${ }^{26}$ (porque estamos muy cristianizados). Ya no hacemos rogativo con fe, falta mucha comunicación entre peñi, entre nosotros mismos, por eso diosito no nos escucha cuando le pedimos..." (Diálogo grupal-Llarquenco Alto).

25. "Awinkados, rume wigkaukvleiñ" son dos conceptos que se pueden traducir indistintamente como "asumir un estilo de vida occidental, es decir, ser como cualquier chileno; pero en este caso cuando se está hablando de la propia tradición religiosa es más correcto traducirlo como "estamos demasiado cristianizados".

26. El conflicto entre cristianismo y religión mapuche se aborda en el libro La religión de la tierra. Una herencia de nuestros antepasados. Reflexiones que en algún momento habria que profundizar. 
Aprendizaje de la vida en nuestra condición de hijos de la tierra

Cuando el mapuche se pregunta acerca de su humanidad, de su ser persona, no solo se pregunta ¿qué soy o quién soy?, sino en relación a ¿quienes soy? Porque él sabe que no es un individuo aislado, sino que él es en cuanto miembro de una familia terrenal y en cuanto miembro de una comunidad humana con una historia particular. En este sentido él es siempre en relación con otros. Estas ideas están presentes en el tuun, el kvpal y el az del ce y el az del mapu ${ }^{27}$.

El tuun hace referencia al lugar de origen, a la tierra, a la geografía y a la historia del lugar; el kvpal se refiere a los vínculos de sangre con los antepasados, en cierta forma se refiere al linaje de la persona; el az se refiere a la identidad, el carácter, la forma de ser del ce y del mapu, porque no solo el ser humano tiene $a z$, la tierra también tiene su forma de ser. Estos conceptos nos permiten entender otra dimensión del ser humano, en cuanto hombre y mujer.

La idea de ser ce (gente, persona, ser humano) está íntimamente relacionada con el mapu (la tierra), porque el mapu es como la matriz, de donde han surgido todos los seres vivos ( $f i j$ mogen), entre los cuales está el ser humano. En este sentido el tuwvn original del ce es el mapu. Esta idea se expresa con el concepto maputuwvn, es decir, procedemos de la tierra, hemos nacido de la tierra en cuanto materia originaria, pero también pertenecemos a un lugar geográfico, a una identidad territorial, a un territorio. En este sentido el maputuwvn da una identidad, un carácter $(a z)$ y una forma de ser persona en relación a la geografía del lugar, es decir, en relación a la forma de la tierra y en relación a la naturaleza en general, en cuanto flora y fauna.
En cuanto a la geografía, podemos decir que la dimensión espacial, la geografía, imprime un rostro, una manera de ser, incluso unas costumbres propias del lugar. Por eso, no es lo mismo ser de la cordillera (pewence) que ser de la costa (lafkence) o de los llanos (nagce), porque la tierra, el territorio en cuanto lugar de origen nos marca, deja una huella en nuestra forma de hablar, en la vestimenta, especialmente en los colores de la ropa, en la elaboración de los instrumentos y en la forma de trabajo. Respecto al idioma (mapucezugun), por ejemplo, no se habla de la misma forma en todo el territorio mapuche, hay variaciones dialectales, es decir, cada zona tiene una forma de hablar que es resultado de la geografía y de la historia del lugar ${ }^{28}$. En otras palabras, es la naturaleza que permite un cierto tipo de lenguaje y una manera particular de vestir ${ }^{29}$. Los colores de la ropa, la ornamentación, el diseño de la vestimenta y de la artesanía pasan a formar parte del carácter y la identidad. En este sentido, el contexto natural influye en la conformación del carácter, lo cual se aprecia en la diferencia que existe entre las distintas identidades territoriales (pewence, lafkence, wijice, nagce, etc.).

En relación al vínculo con los elementos de la naturaleza, el maputuun vincula también con animales, aves, reptiles, y esto se expresa en el significado que tienen los nombres originales, que con el transcurso de la historia y especificamente durante el proceso de chilenización, se transformaron en apellidos mapuches. Originalmente cada persona en cuanto individuo, para distinguirse de los demás solo tenía un nombre (gvi) que lo identificaba. Estos nombres tenian que ver con ciertas cualidades que manifestaba la persona, en la medida que iba creciendo o bien los nombres hacían referencia a las cualidades de los seres vivos residentes en el lugar y estos podian ser animales, serpientes,

27 El az mapu se refiere a las normas juridicas, morales, sociales y culturales que regulan la convivencia entre los seres vivos.

28 En la zona de Tirúa por ejemplo no pronuncian la "f" para decir "feula o faupvle", dicen "veula y vaupvle".

29 Los adornos de la mujer mapuche en la zona de Lumaco y los alrededores es diferente a como se viste y adorna la mujer en la zona de Nueva Imperial. 
aves, etc. Dichos nombres eran vinculantes, en el sentido que tenían la capacidad de establecer comunión, algo asi como un traspaso de energía, que permitía apropiarse de las cualidades de dicho animal. Existen, por ejemplo, nombres relacionados con el pangi, el magke, el gvrv, el filu, etc. (puma, cóndor, zorro, serpiente). Todos estos nombres hacen referencia a las cualidades que distinguen a estos seres vivos y la idea es que esas cualidades se vean reflejadas en la persona. Dicho de otra manera, los nombres mapuche responden a su kvpal, es decir, al comportamiento y a las cualidades de ese animal. Dicho animal le da al mismo tiempo un $a z$, una identidad, un carácter y una forma de ser.

De esta forma el maputuwvn permite identificar y conocer al ce:

"Xekan meu, zugun meu, ayvn meu, kvzau meu, tukutun meu, kimgekei ta mapuce wenxu, mapuce zomo"... (Orfelina Zuñiga-Kayurankil).

"En el caminar, en el hablar, en el reir, en la forma de trabajar, en la forma de vestir se conoce al hombre y a la mujer mapuche..."

La otra dimensión del kvpal se refiere a las relaciones de sangre, es decir, la vinculación con sus antepasados, con su linaje ancestral. En este sentido el kvpal hace referencia a un carisma o a una cualidad de algunos de los antepasados. Dichas cualidades humanas tienen que ver con ciertos valores como: el respeto (yamuwvn), la justicia (nor zugu), la sabiduría (kimvn), las habilidades en general, como por ejemplo, la habilidad discursiva (weupife), la habilidad deportiva (palife), la habilidad guerrera (aukafe o weicafe), etc.

De esta forma, cuando una persona se presenta ante los demás, junto con dar a conocer su tuwvn, habla también de su kvpal que lo relaciona o vincula directamente con sus antepasados:

"Kuifi tañi frcece ka tañi lakuyem palikefuigvn, ka lof magelkefuigvn, fei rupan aukantulu-egvn, micaukefuigvniael, kicuken cajakorv elukefuigvn ñi kon, wigka ta convivencia pi, fei femkefuigvn kuifi...
Palin mu mvlekefui xuxukatun, pifijkatun, kugkujtun...quebramu kintukefuigvn lolkiñ de cardo fei wirar-rumekefuigvn... Kom lof mu mvlekefui palin, cajakorv misau-i-egvn aukantunmuyemai. Ince fí cau mvna kvme palife-fel, feimu ince zugun, meu nvxam feipiken, palifekvpal ta ince..." (Domingo Coña-Pillummallin).

"Antes mi papá y mis tíos jugaban a la chueca, invitaban a otra comunidad y al terminar el juego, compartian la comida, cada palife atendia a su kon con una olla de comida. Los wigkas dicen convivencia. Asi era antes. En los juegos de palin se tocaba la xuxuka, la pifijka y el kugkuj... en las quebradas buscaban cardo y con eso hacian un instrumento que se llamaba lolkiñ y lo hacian sonar. En todas las comunidades jugaban al palin y eso era un motivo para compartir la comida. Mi padre era un gran palife por eso, yo cuando hablo o en una conversación siempre digo con orgullo: yo procedo de grandes palife...".

Reivindicar el kvpal no es otra cosa que reivindicar una determinada categoría social de pertenencia, que tiene que ver con un rango, que podría ser equiparado al orgullo familiar, en relación a otros que son solo "re ce" (gente común). Pero este rango social no es sinónimo de privilegio, sino más bien se trata de un don, un carisma en cuanto posibilidad de ser. Es decir, si yo procedo de grandes y sabios logko, la comunidad espera que yo herede ese don o esa cualidad y lo ponga al servicio de la comunidad. Esta es la razón principal por qué de un hijo de logko se espera que sea el sucesor de su padre, asi también del hijo de un weupife (sabio y conocedor de la historia) se espera un futuro weupife, de los hijos de un maci o una maci se espera que alguien de la familia herede ese don.

El kuifi (el tiempo pasado) iluminador del ser mapuche hoy (faci antv, feula, fau)

Los cambios que ha experimentado la sociedad mapuche, en todos los ámbitos de la vida, plantea el desafío de una renovación espiritual a partir de los propios símbolos y valores. Los ancianos/as (fvcakece) repiten insistentemente: "feula rume kalei taiñ mogen", haciendo referencia a que la vida 
ha cambiado excesivamente y que hay algo "extraño - raro" que no calza con nuestra forma de vida original. Esta percepción de "rareza" en nuestra vida, parafraseando al filósofo Ricardo Salas ${ }^{30}$, sería consecuencia de una transformación de nuestros imaginarios simbólicos tradicionales, que lleva consigo la búsqueda de un sentido de la existencia a partir de lo propio.

\section{a) Ser persona con valores}

Así como un árbol, desde el momento que deja de ser semilla, es la tierra que le da todas las posibilidades para alcanzar su madurez, de la misma forma el ser humano recibe la fuerza y la energía ${ }^{31}$ de la tierra y del cosmos para lograr su plenitud y diferenciarse del resto de los seres vivos. En esta situación el ser humano se va haciendo en el tiempo de acuerdo a un ideal de persona y en este hacerse en el tiempo aquí y ahora, el kuifi, es decir, el "antes", no solo como recuerdo de un tiempo pasado y de una mejor vida, juega un papel importante en la construcción del presente, del aquí y ahora (fau, feula). En este sentido, el pasado no se puede olvidar, porque sin un pasado no hay un presente. Hoy, después de todos los cambios vividos, es necesario volver a recuperar aquellos valores fundamentales que en algún momento impregnaron la vida mapuche en el pasado.

De acuerdo a la enseñanza tradicional, los principales valores que debe aprender toda persona son los siguientes: el respeto (yamuwvn), la solidaridad (keyuwvn), la rectitud en el actuar (nor piuke) y un pensamiento que respete la vida (kvme rakizuam), entre otros.

"...Los fvcakece (viejitos) sabian aconsejar a las personas, gvlam dicen los mapuches... Mi abuelita me aconsejaba y me decia: usted debe ser una persona buena (kvme ce), respetuosa... Deben ayudarse entre vecinos, entre familiares... Nos decian también: no se metan en cosas malas (weza zugu). No roben. No tomen lo que no es de ustedes... los viejitos son gente de experiencia, saben dar consejos, cada cosa que ellos dicen hay que tomarlas en serio..." (Diálogo grupal-Maiten Oñoico-).

"Kuifi zoi mvlekefui keyuwvn, zoi poyeukefui ce, wixankontu-ui, pentukuui, micau-i. Ilosancuyekefui ñi cau fei ka yekefui ñi prima mu ñi ñukentu mu amukefui ka yekefui yekefui we kexan... Rume kvme ce mvlekefui kuifi, feula mai gewelai femgeci ce, mvte micaulai ce, cada uno en su casa nomás... Kuifi ilozancui ce, moxiltu kom vecino mvlepui fei niewetukelafui ilo lagvmzanculu, kom micaukefui-em..

Welu feula epe-gewelai feici zugu, wigka ñi zuam piken ta ince... Welu ka picin mvlei gvxirtuwvn, feikam feula nielu ce, kuifi nielafui pobregei weluke-keyuwi ta ce welu feula zoi nielu zoi femgelai, porque feitiga niei keyulayafiiñ kagelu ka plata pikei. Kuifi femlafui ta ce, weluniei kvzau wilu-wilugei ce, mingako pigei, en el dia pvxvn ichona kompui feula ta femwelai-ce, nielu plata fei kuji, nienulu plata makina kintui-ka fei mu mekei...

Kuifi mai rume kvme gvlam elukefuigvn pu fvcakece, rukamu, matetun meu nvxamkakefuigvn"... (René TripainaoKayurankil).

"Antes la gente se ayudaba más, se querian más, se visitaban y hacian el pentuku, para ponerse al tanto de la situación de toda su familia y comunidad y compartian comiendo de un mismo plato. Cuando mi papá mataba un chanchito le llevaba carne y trigo nuevo a su ñuke...

30 Ricardo Salas, en su libro Lo sagrado y lo humano, plantea algunas reflexiones relativas al simbolismo de las culturas tradicionales... donde demuestra cómo los símbolos religiosos van rearticulando un mundo sagrado a partir de las propiedades del lenguaje simbólico, p. 13.

${ }^{31}$ Si comemos una manzana no comemos simplemente una manzana. Cada manzana es tierra, es energía solar, es la concentración de todas las energías cósmicas. 
Antes había gente buena, ahora ya no hay esa calidad de persona, ya no se comparte, sino que cada uno en lo suyo... Antes cuando se mataba un cerdo, un moxiltu, iban casi todos los vecinos y el dueño de casa prácticamente quedaba sin carne ya que todo se compartia...

Ahora como que se está terminando esa forma de vivir, somos más desunidos, por culpa de los wigka, digo yo. Aunque también hay envidia y divisiones internas entre nosotros, puede ser también porque ahora tenemos una mejor situación económica. Antes éramos más pobres, pero nos ayudábamos unos con otros, en cambio ahora al que tiene ya no se le ayuda. Ahora se ayuda por plata. Antes no era así, cuando alguien tenía trabajo todos iban a ayudar, eso se llamaba mingako, si habia que cortar trigo todo el mundo llevaba una pichona. Ahora ya no. Ahora hay que pagar o si no buscar una máquina... Antes los antiguos sabian dar gvlam (consejos) y lo hacian en la casa, tomando mate y conversando...".

En el imaginario de los kimce (sabios), el kuifi, en cuanto representa una experiencia de vida acumulada, era una época de una vida simple, sin grandes preocupaciones, con más tiempo para vivir. En ese tiempo "la gente era mejor, se visitaban y se ayudaban más, ahora ya no hay esa calidad de persona". En cambio el fau, feula, se refiere al tiempo presente, aunque tenemos más comodidades, tenemos al mismo tiempo más preocupaciones y menos tiempo para compartir. Se trata de un estilo de vida donde el hombre vive para trabajar y ya no hay tiempo para compartir. En esta situación el pasado es iluminador. El saber vivir, el vivir una vida buena definitivamente supone un ideal de ser humano que se encuentra en el kuifi.

Por lo tanto, ¿cómo debemos ser y cómo debemos vivir la vida? De acuerdo a la experiencia debemos ser gente con valores, seres humanos con un "nor piuke" y un "kvme rakizuam". Es decir, un corazón recto, sincero y un buen pensamiento o un pensamiento constructivo que genere y respete la vida. En este sentido sería un pensamiento vital, un pensamiento de vida. Por eso cuando un lider da un mensaje a su comunidad, literalmente le pide encarecidamente que dicho mensaje se haga cabeza y corazón, es decir, se haga pensamiento vital y sentimiento; que se haga carne, sangre y hueso:

"Tvfaci zugu, tvfaci nvxam piukeluupe pu peñi pu lamgen, logkoluupe, foroluupe feimu mai kvme amuai taiñ zugu..." (Domingo Coña-Pillumallin).

"Estas palabras, este mensaje deseo que se grabe en sus corazones, hermanos y hermanas, que se grabe en su mente (pensamiento - razón), que se haga hueso... que se haga carne, es decir, que se haga vida para que lo nuestro permanezca en el tiempo..."

\section{b) La vida en la otra tierra (ka mapu...32)}

De lo que es posible extraer de los nvxam (diálogo grupal e individual), es que toda persona en cuanto individuo, en su contextura existencial, es mapu y pvjv (tierraespíritu). El haber nacido de la tierra significa ser tierra, ser materia. La materia representa el cambio permanente, la capacidad de comenzar de nuevo. En el ser mapuche la tierra representa también la inmanencia, el ser de aquí, el existir aquí y ahora, el tener una existencia mortal. En cambio el pvjv representa la trascendencia, la continuidad de la vida en otra tierra ( $k a$ mapu). Por eso, de acuerdo a la sabiduria tradicional, al difunto se le despide con discursos y se le prepara un "equipaje para el camino", que consiste en alimentos, bebida y ropa de vestir que se deposita en el sepulcro, porque existe el convencimiento de que la vida continúa en el más allá, en la otra tierra.

32 El prefijo "ka" unido a "mapu" (kamapu) da el sentido de distancia, lejanía (por ejemplo, "kamapu amui kvzau-al tañi inan peñi - lejos se ha ido a trabajar mi hermano menor"). En cambio cuando el prefijo "ka" va separado de "mapu" (ka mapu) da el sentido de otra tierra, otro lugar. 
Estas ideas aparecen principalmente en los nvxam y en un contexto de despedida al difunto (eluwvn), donde es posible ver también una cercanía con el pensamiento cristiano, con la diferencia que la vida en el más allá no es resultado de un premio por un buen comportamiento en la tierra. Para los fvcakece no existe una vida en el más allá, como un lugar de gozo o como un lugar de sufrimiento eterno. Esa otra tierra es el lugar de los espíritus y en ese sentido no es sinónimo de "cielo" cristiano.

En el imaginario mapuche el "ka mapu" representa la continuidad de la vida. Quizá esta sea la dimensión de la vida mapuche que ha incorporado más elementos simbólicos venidos del cristianismo.

"Kuifi la-lu fvcakece el-femgelai, kuifi kimgelai urna, wuampo tati, pijin meu zeumagekefui, kiñe semana pigei mekegei ñi zeumagen ti urna, feiti wuampo fei ka tukugei puro pellin nomas ka tapanakvmgei, fei-ula elgekefui, piam. Fei mareupulgei pun ka antv fei fvxa eluwunmagei. Femgeci femkefui ce, ince pici kimpan ñi abuelo la-lu picizomogen. Kimtun ka kimwetukelan fvta lomamu elgeí tañi fvca cau-em, mvlei fau cordiontumekei ce, pali paligei, kaweju plata mvlei wajomekei feimu kom mareupul mekegei awunmagei..." (Orfelina Zúñiga - Kayurankil).

"Antes cuando moría un anciano no lo sepultaban inmediatamente, incluso no se conocía la urna sino que buscaban un tronco de pellín y ahí solian demorar una semana en hacer el wampo (batea) y una tapa, todo de pellín y ahí ponian al difunto.

Hecho estos preparativos hacian el mareupul $^{33}$ (rito de despedida) tanto de noche como de día, a continuación se hacía el eluwvn (comida de despedida). Asi hacian antes, yo alcancé a conocer eso cuando murió mi abuelo, yo era una niña pequeña. Tengo así como un recuerdo-como un sueño- que cuando murió mi abuelo, lo sacaron a la loma, la gente tocaba la acordeón, otros jugaban al palín, otros con sus caballos adornados de plata deban vueltas alrededor del difunto haciendo el awvn...".

"... La, tukulgekefui urnameu muzai, pulku, kankan-ilo gvñvnuam, feipikefui ce. Welu kiñeke mu pu fvcakece ka feipikefuigvn lalu-ce mapu-utukei, welu ñi pvjv kañpvle amutukei, ka mapu..." (Florencio Tripainao - Kayurankil).

"... Al difunto, le ponian en la urna mudai, vino, asado, para que no pase hambre, decian. Pero algunas personas de edad también decian que cuando uno muere se vuelve tierra pero su pvjv (espiritu) se va a otra parte, a otra tierra...".

Estas convicciones religiosas han permitido incorporar, sobre todo en las nuevas generaciones mapuches, las nociones cristianas de cielo e infierno, como resultado de un premio o castigo después de la muerte.

\section{A manera de conclusión}

Las sociedades latinoamericanas, incluyendo las sociedades indígenas, hoy son mayoritariamente cristianas, se trata de un cristianismo en crisis que ya no es capaz de orientar la vida de las personas para una mejor vida. Lo que podemos ver diariamente en nuestro país, a través de los diversos medios de comunicación, prensa escrita y televisión, es claramente una inversión de los valores. Por nombrar algunos aspectos más relevantes, hoy lo que importa es tener y disfrutar la vida, sin importar los medios utilizados para el logro de esos objetivos. Los asaltos y los

33 Mareupul es un rito solemne para despedir al difunto; el amulpvjvn es un acto en que los dolientes cercanos se dividen en dos grupos y se acercan a la urna: los familiares directos por un lado y los cuñados al otro lado. Se ganan en dos filas frente a frente y la urna al centro, enseguida hablan sobre la vida del difunto. Cada cierto tiempo toman un poco de vino intercambiando las botellas. El awvn se realiza antes de llevar el difunto al cementerio y consiste en lo siguiente: mientras los dolientes, amigos y familiares hacen guardia al difunto, un grupo de personas con sus caballos adornados y tocando instrumentos dan vuelta alrededor del difunto. 
crimenes muestran claramente un desprecio a la vida. Por otro lado, a pesar de que estamos cada vez más comunicados, estamos al mismo tiempo más solos y aislados, porque el estilo de vida moderno es demasiado individualista.

Sin embargo, el anhelo de una vida buena, sin duda está presente en cada ciudadano chileno y en cada mapuche, y para hacer realidad este deseo de una vida mejor, el kuifi mogen mapuce, es decir, la tradición y el pasado, en cuanto experiencia de vida, con un respaldo de miles de años, tiene un ideal de persona para estos tiempos de crisis. Algunas caracteristicas de este nuevo ideal de ser humano son las siguientes: primero, respetar y cuidar la Madre Tierra porque ella nos alimenta y sostiene. Es la tierra que nos permite ser y existir. Pertenecemos a la tierra, la tierra no nos pertenece. Esta convicción es la principal razón de por qué debemos manifestarle respeto y cariño (reverencia) a la tierra. A la madre se cuida y respeta. El culto a la tierra no es una deificación. Rendirle culto a la tierra es cuidarla y respetarla, es reconocer que dependemos de ella; segundo, el mito del Xen Xen y Kai Kai filu nos recuerda dos verdades fundamentales: la naturaleza castiga, manifiesta su enojo y eso lo podemos ver claramente con los cambios climáticos que estamos experimentando, hecho que se debe entender como un urgente llamado de atención a un cambio de mentalidad en el trato que damos a la naturaleza, y la otra verdad tiene que ver con reconocer y valorar el hecho de que la Madre Tierra es productora de vida. Ella es el origen y madre de la diversidad (fij mogen mvlei taci mapu meu). Todos los seres vivos dependemos de ella; tercero, en este existir aqui y ahora ( $f e u, f e u l a)$ el pasado (kuifi) es iluminador del presente. El pasado en cuanto recuerdo de una vida sencilla es una vida con valores. Este aprendizaje de la vida permite sentirse parte de la tierra y de la naturaleza en general y al mismo tiempo pone los límites a la libertad del ser humano, porque el hombre no puede hacer todo to que lé de gusto y gana en la Madre Tierra; y finalmente un cuarto aspecto a recordar, aunque somos del mundo, no somos amos ni señores del mundo. Esto significa aceptar los limites de la naturaleza humana y respetar los derechos de otras formas de vida en esta casa común, que es la Madre Tierra (Ñuke Mapu).
Bibliografia

ANTILLANCA, ARIEL Y LONCON, CÉSAR. EsCritos mapuches, 1910-1999. Publicado por Consejo Nacional del Libro y la Lectura y Asociación Mapuche Xawun Ruka.

CURIVIL PAILLAVIL, RAMÓN FRANCISCO. LOS cambios Culturales y procesos de re-etnificación entre los mapuches urbanos. Un Estudio de Caso. Comuna de Pudahuel, Santiago (Tesis de Magister, Universidad Academia de Humanismo Cristiano, 1999).

La fuerza de la religión de la tierra. Una herencia de nuestros antepasados. Publicación financiada por Institut Missio Aachen, Alemania. Ediciones Universidad Católica Raúl Silva Henriquez. Santiago, 2007.

DÍAZ FERNÁNDEZ, JOSÉ FERNANDO. El proyecto histórico del Pueblo Mapuche. Unas lectura misionológica desde un horizonte no sacrificial (Tesis de Doctorado Pontificia Universidad de Teología, Nuestra Señora de la Asunción, San Pablo, Brasil, 2000).

DESCARTES, RENÉ. Discurso del Método. Prólogo y cronología de Mauro Armiño. EDAF, Ediciones - Distribuciones, S. A. España, Madrid, 1982.

FORNET-BETANCOURT, RAÚL. Transformación intercultural de la filosofía. Palimpsesto Derechos humanos y Desarrollo. Editorial Desclée de Brouwe, S.A. Bilbao, España, 2001.

Concepciones del ser humano e interculturalidad. Culturas de humanización y reconocimiento. Concordia. Internationale Zeitschrift für Philosophie. Reihe Monographien / Serie Monografia. Tomo 48. Gedruckt mit Unterstützung des Missionswissenschaftlichen Instituts Missio e.V (MWI), Aachen.

El lugar de la tierra en las culturas. Un diálogo de cosmologías ante el desafio ecológico. Dokumentation des XIII. Internationalen Seminars des Dialogprogramms Nord-Süd. Wissenschaftsverlag Mainz 2009.

MINISTERIO DE EDUCACIÓN, DIVISIÓN DE EDUCACIÓN GENERAL, PROGRAMA DE EDUCACIÓN INTERCULTURAL BILINGÜE. Análisis de los contenidos de los libros de texto de educación básica desde la perspectiva de la diversidad cultural. Santiago, enero de 2001. 


\section{Revista CUHSO volumen $16 \mathrm{~N}^{\circ} 2$}

SALAS ASTRAIN, RICARDO. Lo Sagrado y lo humano. Para una hermenéutica de los símbolos religiosos. Editorial San Pablo, Santiago de Chile, 1996.

"Religiones, filosofía inculturada e identidad (pistas para pensar la religión en la modernidad avanzada)", en Boletín de Filosofia $N^{\circ} 10,1999$, pp. 183-206. Universidad Católica Cardenal Raúl Silva Henríquez.

"Para una reconstrucción intercultural de la historia republicana" (Artículo), en: Universum. Revista de Humanidades y Ciencias Sociales. VIII Corredor de las ideas del Cono Sur. Talca, 3 y 6 de enero del 2007

SENENT DE FRUTOS JUAN ANTONIO. "La tierra y la naturaleza en el horizonte de la subjetividad moderna". Ponencia presentada en Seminario Internacional sobre Filosofía de la tierra. Barcelona, España. Publicado en: El lugar de la tierra en las culturas. Un diálogo de cosmologías ante el desafío ecológico. Dokumentation des XIII. Internationalen Seminars des Dialogprogramms Nord-Süd. Wissenschaftsverlag Mainz 2009 (páginas 163 - 183).

TAYLOR CHARLES (ENSAYO). El multiculturalismo y la politica del reconocimiento. Princeton University Press, 1992. Traducido por Mónica Utrilla de Neira. Colección Fondo de Cultura Económica, México.

\section{Revistas}

Boletín de Filosofía, $N^{\circ}$ 9, vol. 3, UCBC, Santiago, 1997-1998.

Revista Pastoral Popular. Año 51 - N ${ }^{\circ}$ 269, Ediciones Rehue, Santiago. Mayo/junio de 2000. 